\title{
Properties of Transition Metals and Their Compounds at Extreme Conditions
}

\author{
Simone Anzellini ${ }^{1, *(D)}$ and Daniel Errandonea ${ }^{2}$ (D) \\ 1 Diamond Light Source Ltd., Harwell Science \& Innovation Campus, Diamond House, Didcot OX11 0DE, UK \\ 2 Matter at High Pressure (MALTA) Consolider Team, Departamento de Física Aplicada-Instituto de Ciencia de \\ Materiales, Universidad de Valencia, Edificio de Investigación, C/Dr. Moliner 50, Burjassot, \\ 46100 Valencia, Spain; Daniel.Errandonea@uv.es \\ * Correspondence: simone.anzellini@diamond.ac.uk
}

Citation: Anzellini, S.; Errandonea, D. Properties of Transition Metals and Their Compounds at Extreme Conditions. Crystals 2021, 11, 1185. https://doi.org/10.3390/cryst11101185

Received: 22 September 2021 Accepted: 22 September 2021 Published: 29 September 2021

Publisher's Note: MDPI stays neutral with regard to jurisdictional claims in published maps and institutional affiliations.

Copyright: (c) 2021 by the authors. Licensee MDPI, Basel, Switzerland. This article is an open access article distributed under the terms and conditions of the Creative Commons Attribution (CC BY) license (https:/ / creativecommons.org/licenses/by/ $4.0 /)$.
The characterisation of the physical and chemical properties of transition metals and their compounds under extreme conditions of pressure and temperature has always attracted the interest of a wide scientific community. Their properties have numerous implications in fields ranging from solid-state physics, chemistry and materials science to Earth and planetary science.

In the last few decades, thanks to advancements in experimental techniques and computer simulations, the rate of new important discoveries in this field has significantly increased: from the prediction and the experimental observation of new ultra-hard materials to the combined characterisation of textural, structural, magnetic and chemical pressure-induced evolutions, and from the possible observation of topological transitions in the Fermi surface for valence electrons to newly predicted pressure-induced core level crossing transitions.

This Special Issue collects twelve contributions, starting with the paper of Minikayev et al. [1]. In this work the authors combine low temperature and high pressure to obtain detailed quantitative information on the evolution of the thermostructural and elastic properties of rock-salt-type crystals of $\mathrm{PbTe}$ and $\mathrm{Pb}_{0.884} \mathrm{Cd}_{0.116} \mathrm{Te}$. The authors found a consistent image of influence on the partial substitution of $\mathrm{Pb}$ ions by $\mathrm{Cd}$ ions in the thermostructural properties of the PbTe lattice. Namely, the authors show how the lattice parameters, the thermal expansion coefficient and other thermostructural properties depend on the Cd content.

The following paper by Bettina Camin and Maximiliam Gille [2] is an interesting example of the direct application an "extreme conditions" research can have to everyday life tools. As a matter of fact, in the race towards a World with zero emissions, lightweight constructions and materials offer the opportunity to reduce $\mathrm{CO}_{2}$ emission in the transport sector. However, the various components in vehicles are often exposed to temperatures that can be up to $40 \%$ higher than their melting temperatures. Therefore there is a risk of creep. In this paper, the authors are comparing the creep behaviour of hot extruded and heat treated ME21 magnesium alloy in both standardised miniature and standardised specimens. The obtained results show evidence of size effects in the creep parameters.

In the article by Littleton et al. [3], the authors report a characterisation of the electrical resistivity of solid and liquid Fe-FeS up to $5 \mathrm{GPa}$ and the corresponding thermal conductivity. From the measured sharp changes in the measured electrical resistivity, the authors managed to delineate the eutectic temperatures of the studied Fe-FeS system as a function of pressure. The combination of the obtained results with thermal models provided an adiabatic heat flow of molten Fe-FeS eutectic composition indicating the possible presence of thermal convection in Fe-FeS under these pressure and temperature conditions. The obtained results provide important information for understanding the dynamo and thermal evolution of the core of Ganymede (the only moon in the solar system with its own magnetic field). 
The characterisation of the pressure and temperature-induced modification of the properties of metallic alloys, such as morphology and microstructure, is extremely important for several technological applications. In the article by Zhang et al. [4], the authors probed the microstructural changes in the Si phase of the Al-20Si alloy at different pressure and temperature conditions, combining a multi-anvil apparatus and Scanning Electron Microscope images. In another original article, Baty et al. [5] performed an ab initio characterisation of the high pressure-high temperature phase diagram of copper. The results, obtained from a quantum molecular dynamic simulation based on the so called $\mathrm{Z}$ methodology, confirm the polymorphic nature of copper. The authors also discuss the consequent reliability of copper as a pressure standard for shock experiments.

In the subsequent contribution, Anzellini et al. [6] reported the first experimental characterisation of the phase diagram of iridium obtained with a combination of a laserheated diamond anvil cell with synchrotron $X$-ray diffraction and density-functional theory calculations. Together with the solid and liquid phase boundary of iridium, the authors also report the corresponding thermal equation of state and compare it with the one of other transition metals.

In the next original contribution, Schiesaro et al. [7] characterise the pressure-induced modification of the local atomic structure of a $\mathrm{Nb}_{3} \mathrm{Sn}$ superconductor. In particular, combining the standard analysis for X-ray absorption spectroscopy data with an evolutionary reverse Monte Carlo modelling, they evidenced a complex evolution in the $\mathrm{Nb}$ chains at the local atomic scale. The reported local effect appears to be correlated to anomalies evidenced by $\mathrm{X}$-ray diffraction in other superconductors belonging to the same family.

In the following work by Mostafa et al. [8], the authors characterised the mechanical properties of a commercial purity aluminium modified with $\mathrm{Zr}$ micro-additive. The obtained results show a Zr-induced reduction in the grain size of the commercial purity aluminium, associated with an improvement of the microhardness number and resistance to fracture, as well as an improved Charpy impact energy and proof stress.

In the article by Liang et al. [9], the authors report the first high-pressure spectroscopy study on $\mathrm{Zn}\left(\mathrm{IO}_{3}\right)_{2}$, a member of the metal iodates, a family of materials characterised by non-linear optical properties. Using a combination of diamond anvil cells and synchrotron far-infrared spectroscopy, the authors observed three phase transitions all characterised by changes in the infrared spectra.

Finally, in the last original work, Diaz-Anichtchenko et al. [10] report a synchrotron $\mathrm{X}$-ray diffraction characterisation of the pressure induced evolution of a Kagome staircase compound $\left(\mathrm{Ni}_{3} \mathrm{~V}_{2} \mathrm{O}_{8}\right)$, belonging to the large family of metal orthovanadates. The experimental results, obtained using diamond anvil cells, evidence an anisotropic response of the orthorombic structure to external compression, with the b-axis showing a compressibility almost $40 \%$ lower than the one of the other two axes. The authors also report a systematic comparison of the bulk moduli of various isomorphic metal orthovanadates.

In addition to the 10 original research articles described above, this Special Issue also include two review articles. In the first one, Tetsuya Komabayashi [11] shows an overview on the recent updates on the phase-relations in Fe-alloys as a function of pressure and temperature. Such phase-relations are of particular interest for the engineering and metallurgy fields-as they serve as recipes for creating specific phases with certain properties-and are of extreme importance for Earth science, as the major components of the Earth's core-subjected to extreme conditions of pressure and temperature-are Fe and a detailed characterisation of the phase domains of their (geophysically relevant) alloys at those extreme conditions can provide important information on the dynamics and evolution of our planet.

The characterisation of the melting line of transition metals under extreme conditions of pressure and temperature is one the most challenging (and debated) subject in the extreme conditions field. In his review paper, Paraskevas Parisiades [12] discusses the main static techniques used for these studies (with a strong focus on the diamond anvil cell), and explores the state-of-the-art in melting detection methods and analyses the 
possible reasons behind the discrepancy observed between melting lines obtained from various groups.

In summary, the articles presented in this Special Issue are a good representation of the broad interest-covering multiple scientific disciplines-in the present topic and they show the latest advancements in the investigation of transition metals under extreme conditions of pressure and temperature.

Acknowledgments: D.E. acknowledges financial support from Spanish Ministerio de Ciencia, Innovación y Universidades (Grants Nos. PID2019-106383GB-C41 and RED2018-102612-T), and Generalitat Valenciana (Prometeo/2018/123 EFIMAT).

Conflicts of Interest: The authors declare no competing interests.

\section{References}

1. Minikayev, R.; Safari, F.; Katrusiak, A.; Szuskiewicz, W.; Szczerbakow, A.; Bell, A.; Dynowska, E.; Paszkowicz, W. Thermostructural and Elastic Properties of PbTe and Pb0.884Cd0.116Te: A Combined Low-Temperature and High-Pressure X-ray Diffraction Study of Cd-Substitution Effects. Crystals 2021, 11, 1063. [CrossRef]

2. Camin, B.; Gille, M. The Effect of Specimen Size and Test Procedure on the Creep Behavior of ME21 Magnesium Alloy. Crystals 2021, 11, 918. [CrossRef]

3. Littleton, J.; Secco, R.; Yong, W. Thermal Convection in the Core of Ganymede Inferred from Liquid Eutectic Fe-FeS Electrical Resistivity at High Pressures. Crystals 2021, 11, 875. [CrossRef]

4. Zhang, R.; Zou, C.; Wei, Z.; Wang, H. Effect of High Pressure and Temperature on the Evolution of Si Phase and Eutectic Spacing in Al-20Si Alloys. Crystals 2021, 11, 705. [CrossRef]

5. Baty, S.; Burakovsky, L.; Errandonea, D. Ab Initio Phase Diagram of Copper. Crystals 2021, 11, 537. [CrossRef]

6. Anzellini, S.; Burakovsky, L.; Turnbull, R.; Bandiello, E.; Errandonea, D. P-V-T Equation of State of Iridium Up to 80 GPa and 3100 K. Crystals 2021, 11, 452. [CrossRef]

7. Schiesaro, I.; Anzellini, S.; Loria, R.; Torchio, R.; Spina, T.; Flükiger, R.; Irifune, T.; Silva, E.; Meneghini, C. Anomalous Behavior in the Atomic Structure of Nb3Sn under High Pressure. Crystals 2021, 11, 331. [CrossRef]

8. Mostafa, A.; Adaileh, W.; Awad, A.; Kilani, A. Mechanical Properties of Commercial Purity Aluminum Modified by Zirconium Micro-Additives. Crystals 2021, 11, 270. [CrossRef]

9. Liang, A.; Turnbull, R.; Bandiello, E.; Yousef, I.; Popescu, C.; Hebboul, Z.; Errandonea, D. High-Pressure Spectroscopy Study of Zn(IO3)2 Using Far-Infrared Synchrotron Radiation. Crystals 2021, 11, 34. [CrossRef]

10. Diaz-Anichtchenko, D.; Turnbull, R.; Bandiello, E.; Anzellini, S.; Errandonea, D. High-Pressure Structural Behavior and Equation of State of Kagome Staircase Compound, Ni3V2O8. Crystals 2020, 11, 910. [CrossRef]

11. Komabayashi, T. Phase Relations of Earth's Core-Forming Materials. Crystals 2021, 11, 581. [CrossRef]

12. Parisiades, P. A Review of the Melting Curves of Transition Metals at High Pressures Using Static Compression Techniques. Crystals 2021, 11, 416. [CrossRef] 